\title{
Anthós
}

\section{Research Proposal for Study: Can Artifical Emulation of Synesthesia Aid Visual Word Recognition?}

Lucas Charles

Portland State University

Follow this and additional works at: https://pdxscholar.library.pdx.edu/anthos

Part of the Psycholinguistics and Neurolinguistics Commons Let us know how access to this document benefits you.

Recommended Citation

Charles, Lucas (2012) "Research Proposal for Study: Can Artifical Emulation of Synesthesia Aid Visual Word Recognition?", Anthós: Vol. 4: Iss. 1, Article 1.

https://doi.org/10.15760/anthos.2012.1

This open access Article is distributed under the terms of the Creative Commons Attribution-NonCommercialShareAlike 4.0 International License (CC BY-NC-SA 4.0). All documents in PDXScholar should meet accessibility standards. If we can make this document more accessible to you, contact our team. 
Research Proposal for Study: Can Artificial Emulation of Synesthesia Aid Visual Word Recognition?

Lucas Charles

Portland State University 


\section{Introduction}

The field of synesthesia has largely been unexplored beyond mere analysis of the condition and only a few papers on the topic of new applications have been published within the last decade. The condition can potentially provide great benefits to the study of the psychology of language along with its role in language use itself. As understanding of this condition continues to grow, one can draw links between it and its effect on language use, thereby enabling a greater understanding of the language process itself. Synesthesia involves the stimulation of one sense along with involuntary activation of another sensory pathway. For those with synesthesia, ninety-two percent of cases are triggered via linguistic inducers, showing deep ties between the pathways used by the condition and those involved with language faculties (Barnett, 2009, p. 1343). This paper will first cover synesthesia along with some of the many permutations the condition takes, then review previous research into the role of synesthesia in psycholinguistics including the subject of improved recall. After providing background, this paper will provide a framework for carrying out artificial simulation of the condition to improve participant's word recognition. Finally, it will attempt to answer the question of what could be done with the results gleaned. The linking of sensory activation, or "cross talk" as it is commonly known, points to new understandings of how humans process information, especially language. Research can also be applied to further investigation of how those without the condition could apply similar techniques to facilitate visual word recognition.

The role of synesthesia has not been adequately researched in light of its relation to lexical decision tasks. It has been previously shown how cognitive research can be effectively 
informed by synesthesia research, yet there are virtually no extant studies on the application of synesthesia along with the possibility of artificial simulation (Kadosh, 2007). This study attempts to address this issue by simulating synesthesia through a period of training; subsequently, the results of the study will provide a baseline for the work involved in future application of synesthesia to other areas.

\section{Background}

Few researchers have previously linked the topic of synesthesia with psycholinguistic research, and fewer still have tried to emulate the effects of the condition. As one of the most fascinating and eye-opening phenomena of modern neuroscience, synesthesia offers a veritable treasure trove of data. Synesthesia is the involuntary experience of one sense with the activation of another; this is automatic and reproducible within those diagnosed with the condition (Pearce, 2007, p. 120). The condition has been linked to several other neurological conditions including eidetic memory and other cognitive effects. According to Pearce, in reference to the widelycited paper on the subject by Baron-Cohen et al. (2009), the prevalence of the condition is close to 1 in 2,000 within the average population; however more recent research has shown the condition to be far more common. Julia Simner, currently one of the most prolific researchers on the subject, has documented the condition as present in 1 in 23 of the population, or four percent (2006a, p. 23). Due to the naturalness and, in the majority of its forms, lack of interference in daily activity, the condition often goes unnoticed for many years, making the cataloging and assessment of its extent difficult (Kadosh, 2007, p. 177). Synesthesia manifests in a variety of 
ways, including Number-Form Synesthesia, which involves spatial arrangement of numbers on a plane; Lexical-Gustatory Synesthesia, involving spoken or written words triggering taste sensations; Sound-Color Synesthesia, involving experiences of color when hearing sounds; or as Ordinal Linguistic Personification, involving assignment of entire distinct personalities to ordered sequences such as days of the week. It is well established that the cross-modal experience of synesthetes is bidirectional, whereupon stimulus works in both directions in the triggering of senses (Pearce, 2007, p. 121; Kadosh, 2007, p. 179; Brang, 2010, p. 170).

As one of the main areas of experimental research involved in synesthesia study, Visual Word Recognition (VWR) plays an important role. This field addresses the perception and processing involved in recognition of the written word. A great deal of research has been conducted on VWR yet there is still disagreement on several aspects of it, namely the mechanisms involved in recognition. Previous research has utilized a number of tools including eye tracking and various kinds of lexical presentation tasks. Two popular lexical tasks include naming tasks in which a participant must name the word presented, and lexical-decision tasks in which a string of letters is shown and a participant must decide if it constitutes a word (Harley, 2008, p. 170). Both tasks implicate several important aspects of VWR, and are demonstrated by using tools such as priming, where material is presented before the target stimulus to affect response, and masking, in which material is obscured to affect response. In this study, a lexical decision task will be administered in an attempt to see whether the structure of color-grapheme synesthesia facilitates VWR. 


\section{Synesthesia's Role in Cognitive Behavior}

In terms of the condition's effect on cognitive behavior, previous work has revealed synesthetes to have increased performance in visual search tasks, as well as both short-term and long-term recall. Work involving synesthesia's role in memory has been inconsistent, however, and in several cases produced conflicting results. Research has tended to agree that synesthesia improves memory, yet the results of researchers such as Smilek (2002) and Rothan (2010) have disagreed on the extent of memory improvement. In its scope, such previous work has pointed to significant improvements in the standard cognitive abilities of non-synesthetes. Since synesthesia is not normally linked to any cognitive deficits, as with the aforementioned difficulty in identifying the condition's prevalence, its role is unique in the positive benefits to several cognitive behaviors with no avert negative side effects. Synesthesia is thus a ripe candidate for emulation.

For visual search tasks involving a grid of graphemes, Ramachandran and Hubbard found performance of synesthetes to be significantly better at recognizing embedded variations on a grapheme-by-grapheme basis (2001a). The researchers produced a numeral grid of randomlyplaced '2's with embedded '5's in various shapes (such as a square) inside the grid. Each grid was presented to the participants and the performance was measured, showing far better recognition in the groupings among synesthetes than the non-synesthetes that were tested (p. 7). In a second experiment the participants were tested with the "crowding effect." Citing Bouma's work on the effect, Ramachandran and Hubbard describe it as the difficulty in identifying an individual grapheme when the target is closely flanked by other graphemes (p. 8). While the 
effect was preserved in synesthetic subjects, they were still able to identify the obscured grapheme by its color response. One participant is recorded to have stated "I can't see that middle letter but it must be an 'O' because it looks blue" (p. 8). The sensory response of synesthetes is then shown to facilitate recognition even under the circumstances of visual obfuscation.

Since Smilek's 2002 study demonstrating the extraordinary memory ability of a twentyone year old participant, the idea of synesthesia's aid to memory has been widely cited (Simner, 2006a, p. 25). The study describes the participant C's ability to memorize large quantities of numbers with a very low error rate, even reciting them back several months later with equivalent accuracy. The effect is explained by C's description of her memorization process, which involved her sensory experiences as a color-grapheme synesthete (p. 548). Smilek describes previous papers linking the idea of memory and synesthesia, including one of the earliest accounts in Luria's 1968 work on the mnemonist "S," standing for Shereshevsky. Shereshevsky was noted for memorizing a 50-digit matrix in three minutes with perfect recall, yet Luria's study was rather problematic due to its lack of empirical data (p. 548). Smilek's analysis and presentation of three similar fifty-digit matrices to participant $\mathrm{C}$ and seven non-synesthetic participants returned confirmatory results, whereupon C's performance with the black-digit matrix and the one congruent with her color associations showed superior recall to all seven non-synesthetic participants. Despite this, her recall of the incongruently colored matrix was incredibly poor at around four percent, compared to the non-synesthetes who scored between eighteen and forty-four percent (p. 549). 
In contrast to Smilek's study, Rothen and Meier conducted a larger and more thorough group study of the memory abilities within a group of synesthetes (2010). The results of Rothen and Meier's research still showed a superior memory yet only within an ordinary range, as opposed to extraordinary advantage (p. 258). Through analysis of forty-four color-grapheme synesthetes, Rothen and Meier found the average performance of participants to score above average in episodic memory tests and extraordinary in visual paired association learning tasks (p. 262). While the results show superior episodic memory, the results are still on average within range of above-average memory and not the extraordinary performance previously shown in research. Furthermore, three of the four short-term memory tests yielded equal results to standard ranges (p. 263). Rothen and Meier reason that the links between memory benefits and synesthesia are not directly related to presence of the condition, but instead to the superior visual imaging abilities of synesthetes. Due to the isolated benefit, however, the condition only provides "islands of ability" (p. 263).

\section{Color-Grapheme Synesthesia}

Of all of its varieties, the neurological condition manifests most commonly in what is known as Color-Grapheme Synesthesia, which constitutes the subject of this study. This form causes synesthetes to associate individual graphemes with specific colors, often specific to shade or level of intensity such as the grapheme "B", which present as a vibrant blue. While the biological conditions that may account for synesthesia are unclear, there has been some linkage to genes due to common appearance among family members (Pearce, 2007, p. 123). 
Furthermore, due to extensive testing along with various accounts of the condition's presence among the blind, synesthesia has been believed to not merely be a learned behavior through childhood, but an inherent condition (Pearce, 2007, p. 124). In Ward's 2006 study, however, rather than dedicated neurological pathways his research with brain imagery has shown that the condition uses many of the same mechanisms involved in non-synesthetes' experience of crossmodal tasks, such as the McGurk Effect (p. 264). This finding hints that further research may allow the effect to be similarly replicable in non-synesthetes. Synesthesia then appears to fall closer to the category of a behavioral condition, rather than a learned or strictly biological behavior.

Color-Grapheme synesthesia, as one of the more common types, has been the nexus of the majority of research into this field, explaining the close ties between orthographical presentation and cognitive processing. This form of synesthesia is also one of the more thoroughly established as it remains consistent with previous psycholinguistic research and the established understanding of reading as involuntary and automatic, as demonstrated with such experiments as John Stroop's well-known Stroop Test (Harley, 2008, p. 178). Research has also pointed to commonalities in color-grapheme synesthesia both cross-linguistically between different speakers and within multilingual speakers themselves. In several cases there have been associations in bilingual speakers across different languages. In one 2009 study conducted by Barnett, Feeney, Gormley, and Newell, the analysis of a bilingual Russian-English synesthete showed commonalities within the lettering scheme between such graphemes as " $\mathrm{R}$ " and " $\mathrm{Y}$ " 
(pronunciations corresponding to [l] and /ja/, respectively). This clearly demonstrates how the associations in color-grapheme synesthesia are form-based rather than phonologically linked (p. 1344).

Several studies have been conducted that seek to measure responses to visual priming experiments. In one such study the participants were primed with pairings contradictory to their stated ones as ascertained via prior data collected. Where one participant might associate black with "G," for example, the experimenters used green instead. In Brang, Edwards, Ramachandran, and Coulson's 2008 study on eight color-grapheme synesthetes, the participants were primed with such phrases as "looking very clear, the lake was the most beautiful hue of...X”, where X corresponded to the associated number or letter of the participant. Responses to the stimuli were recorded via event-related brain potentials with congruous stimuli triggering less negative N400s in synesthetes in response to incongruous stimuli (p. 425). One prompt, and the namesake of the paper, was the phrase "is the sky 2?", where congruity of one participant's association with "2" as blue produced agreeable responses to similar text-based responses (p. 423). While the control non-synesthetes experienced equal response to congruous and incongruous stimuli, the contextual response of the tested synesthetes points to a noticeable effect on VWR in modulating N400 spikes (p. 427).

While previous studies have examined the effects of synesthesia on Visual Word Recognition, little work has been done in its application. One presentation during the proceedings of the 2005 International Symposium of Wearable Computers attempted artificial simulation, but the focus of their work comprised computer hardware emulation with little regard 
for the linguistic aspects of the effects. This study by Plouznikoff (2005) instead focused on performance in visual search tasks in terms of a proof of concept. While it demonstrated interesting results with the eight participants, who showed an average thirty-point-eight percent increase in recall as well as exhibiting all basic characteristics of non-emulated synesthesia (such as involuntary activation and stability of response over time), the experiment was insufficient in several key ways. The demonstration was focused on the computer science and engineering aspects of the application, lacked a cognitive component, and was also somewhat deficient in terms of true scientific rigor. However, in light of the results and previous research on synesthesia, the work showed promise and justification in pursuing further testing on the subject, specifically with regard to the linguistic applications of synesthetic emulation.

\section{Rationale}

This study will address one key question: Does artificial grapheme-color association aid nonsynesthetes in recognition tasks?

As previously addressed, much research on synesthesia has shown greater recognition and recall skills among synesthetes. Whether these cognitive benefits result from synesthesia or are only correlations has not been established. By emulating synesthetic association, this study will attempt to replicate the cognitive effects of synesthesia in non-synesthetes.

If the initial hypothesis is confirmed, the benefits of synesthetic emulation could be applied to a variety of fields, including education and related areas that would greatly benefit from increased VWR. In terms of the psychology of language it would more clearly connect the 
fields of cross-modal interaction and visual search to the role of color in such psycholinguistic tasks as form-based priming, neighborhood effects, and attentionality in processing (such as the mandatory aspects of reading).

If the initial hypothesis is refuted, one could conclude from the research that the cognitive benefits commonly seen with synesthetes are not directly connected to associative colorgrapheme recognition, but perhaps to another factor. Refutation of the hypothesis would also show a disconnect between facilitation of word recognition and visual search tasks, leading to evidence against certain cross-modal interactions.

\section{Method and Design}

In light of research on synesthesia's role in aiding retrieval, this study will utilize the data that has been collected on consistencies in Color-Grapheme Synesthesia in different participants and identify the most commonly linked colors with their graphemes. Lexical decision tasks will be conducted first without modification, and then, after visual training, once again with grapheme coloring added according to the commonality data. The results of the two separate tests will be compared using a standard t-test and should demonstrate whether or not artificial simulation of synesthetic effects improves visual word recognition in tasks.

As previously mentioned, certain commonalities in synesthetic color-grapheme associations have been shown across multiple synesthetes. In Simner's work on color-grapheme synesthesia and association trends $(2006 \mathrm{a}, 25)$ the use of statistical and linguistic analysis enabled her to note three general themes: (a) that high-frequency colors tend to pair with high- 
frequency graphemes, (b) that associations tend to follow initial letter priming in words (' $r$ ' pairs with "red"), and (c) that pairing of high-frequency letters and lower numbers tend to follow the established typology of color words that arise in languages with few to greater color terms. In a previous study in 2005, Simner and her colleagues were able to collect data and test the responses of seventy native English-speaking synesthetes. Drawing from the data revealed in his research, the "prototypical" synesthetic alphabet (Figure 1) will become the basis for emulation within participants. However, the data does present a few problems, such as the lack of representation for the letter "k." In the study, participants felt no strong color association with the letter. In this case we assigned " $\mathrm{k}$ " the default shade of black.

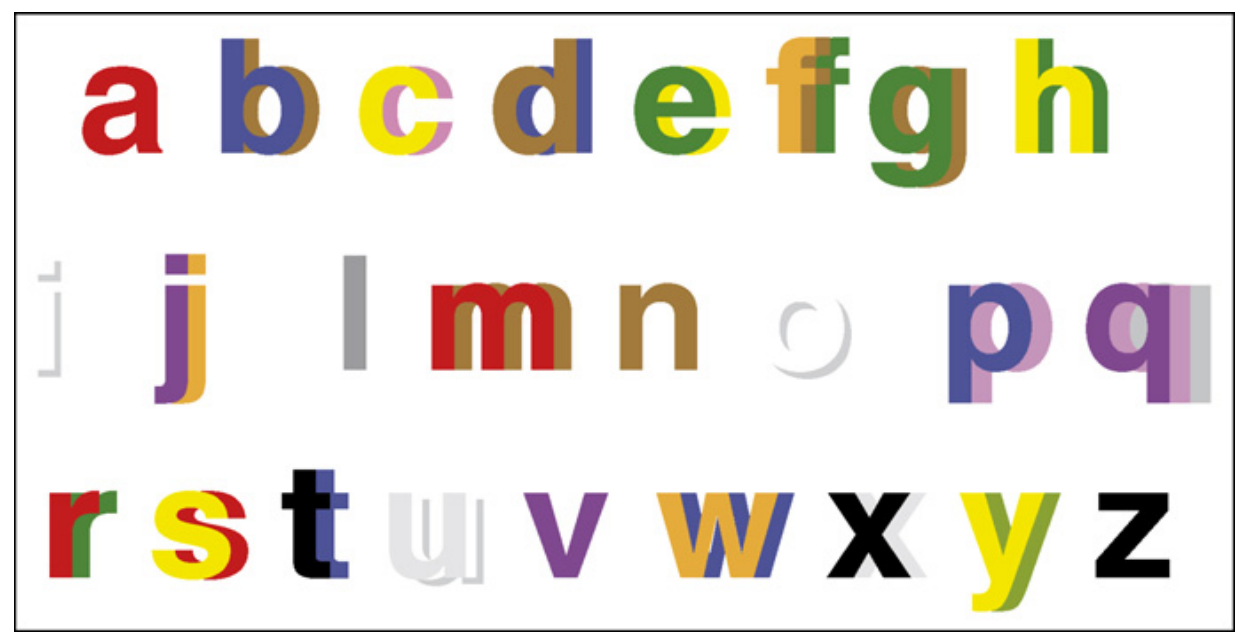

Figure 1. "Representation of the 'prototypical' coloured alphabet of 70 grapheme-colour synaesthetes. Letters in two or more colours represent those with more than one significant shared colour preference; reported colour terms (e.g. red) are represented here as focal colours." Figure retrieved from Simner, 2006a, p. 25. 


\section{Participants}

An adequate sample of individuals for this study should be between thirty to forty healthy adults who are native speakers of English. This would include a distribution of adult males and females, depending on available participants. The group would be of a varied age, ethnicity, and economic status to be representative of the standard US population. Since the test will be conducted using a written text and a computer program requiring some interaction, the participants will be required to have some basic computer proficiency and high school graduate level literacy skills.

All participants will be carefully screened to prevent any predilection toward any synesthetic condition. Participants will be briefed on the condition with presentation of data on the ways in which synesthesia can manifest and asked if they have or currently experience any form of synesthesia and, if so, which one they believe they may have. Any kind of affirmative response will preclude them from the study.

The selected participants will be randomly divided into two groups, with one as the experimental group and the other designated as the control. The participants will not be told to which of these groups they belong. The experimental group will undergo the synesthetic training process between testing while the control group will be used as a comparative baseline to measure the results of the training on the experimental group. 


\section{Design}

Once participants are selected and divided into the two groups, each of them will perform a lexical decision task. This test will be administered via computer with each participant being placed before the program and with the presentation of each lexical item told to respond in one of two ways: by pressing the "Yes" button if the string of graphemes presented on the screen forms a word, or pressing the "No" button if the string of graphemes presented does not form a known word. The testing will be conducted on an individual basis with the participants asked to indicate their decision as quickly as possible. For the two different rounds of testing, a seventytwo word corpus of thirty-six non-words and thirty-six words will be prepared, randomized, and divided into two data sets. The first data set will be administered initially in both participant groups, and the second data set will be administered after synesthetic association training. The response time (RT) for each lexical decision task response will be measured and recorded for analysis. After the first test has been conducted, the participants will be dismissed, and the next test will be conducted approximately one week later.

Between the first and second lexical decision testing there will be a period of training for the group that is to undergo synesthetic emulation. This group will be presented with a prepared collection of newspaper articles to read in which the text provided is colored on a grapheme-bygrapheme basis according to Simner's collected frequency data (2005). Similarly, the control group will be given the same text, but with no coloring. Each participant will be told to read one article per day during the week before they are to return for follow-up testing. The secondary 
lexical decision task will be conducted in an identical manner to the first lexical decision task, but this time using the second grouping of words from the seventy-two word data set in order to prevent repetition of any remembered response.

\section{Analysis}

The data gathered from the initial lexical decision task and the secondary task will be used to measure the effect of the synesthetic training process. The non-synesthetically trained group data will act as a control to measure the synesthetically trained group to show any standard deviations that could result were the secondary round of testing to vary from the initial for any reason. If in the secondary testing participants were to average significantly lower or higher rates of facilitation then this data could be used to adjust the results of both groups accordingly.

The data between the two groups will be compared to confirm the initial hypothesis and determine if the group that underwent synesthetic emulation showed significant improvements in performance over untrained visual word recognition. A standard t-test will be performed to measure differences between the sets and determine if there is any increase in facilitation speed due to the synesthetic training. If after adjustment of the data there is no significant difference compared to the control group then the initial hypothesis will be refuted.

\section{Conclusion}

In consideration of the experiment's results, strides within the fields of both the psychology of language and the greater field of psychology itself would be put in a striking new 
light. With the previous research on synesthetic application being rather insufficient, this study will contribute to a greater understanding of the cross-modal interactions involved in visual word recognition and the process involved in word recognition, as well as the process involved in word recognition. The connection between linguistic inducers and synesthetic response is already well-established, yet the extent of that connection has not yet been fully explored. Furthermore, with the results of the experiment, the application of synesthetic effects to other fields involved in reading and comprehension are widespread and extend beyond an understanding of cognitive processing to a variety of additional research areas. Synesthesia is a fascinating condition with huge potential for further innovative investigation, and this research would mark the first step in opening the doors to a greater psychological and physiological understanding of the brain and its inherent mechanisms of language. 


\section{References}

Barnett, K.J., Feeney, J., Gormley, M., \& Newell, F.N. (2009). An Exploratory study of linguistic-colour associations across languages in multilingual synaesthetes. Quarterly Journal of Experimental Psychology, 62(7) 1343-1355.

Blasko, D. (January 22, 2010). Lexical Decision. Retrieved from http://www.psych.uni.edu/psychexps/instronly_page/leicaldesi.htm

Brang, D., Edwards, L., Ramachandran, V.S., \& Coulson, S. (2008). 'Is the sky 2?’: Contextual Priming in Grapheme-Color Synaesthesia. Psychological Science, 19(5), 421428.

Kadosh, R. (2007). Can Synaesthesia Research Inform Cognitive Science?. Trends in Cognitive Sciences, 11(4), 177-184.

Mills, C.B., Viguers, M.L., Edelson, S.K., Thomas, A.T., Simon-Dack, S.L., Innis, J.A. (2002). The Color of Two Alphabets for a Multilingual Synesthete. Perception, 31(11) 1371 1394.

Pearce, J. (2007). Synaesthesia. European Neurology, 57, 120-124.

Nelson, D. L., McEvoy, C. L., \& Schreiber, T. A. (1998). The University of South Florida word association, rhyme, and word fragment norms. Retrieved from http://www.usf.edu/FreeAssociation/

Plouznikoff, N., Plouznikoff, A., \& Robert, J.M. (2005). Artificial Grapheme Color Synesthesia for Wearable Task Support. ISWC 2005 Proceedings of the Ninth IEEE International Symposium on Wearable Computers. 
Ramachandran, V.S., \& Hubbard, E.M. (2001). Synaesthesia A Window Into Perception, Thought and Language. Journal of Consciousness Studies, 8(12) 3-34.

Rothen, N., and Meier, B. (2010). Grapheme-Colour Synaesthesia Yields an Ordinary Rather than Extraordinary Memory Advantage: Evidence from a Group Study. Memory, 18(3), 258.

Smilek D., Dixon, M. J., Cudahy, C., and Merikle, P. M. (2002). Synesthetic Color Experiences Influence Memory. Psychological Science, 13(6), 548-552.

Simner, J., Ward, J., Lanz, M., Jansari, A., Noonan, K., Glover, L., \& Oakley, D. A. (2005). Non-Random Associations of Graphemes to Colours in Synaesthetic and NonSynaesthetic Populations. Cognitive Neuropsychology, 22(8), 1069-1085.

Simner, J. (2006a). Beyond Perception: Synesthesia as a Psycholinguistic Phenomenon. Trends in Cognitive Science, 11(1), 23-29.

Simner, J., Glover, L., \& Mowat, A. (2006b). Linguistic Determinants of Word Colouring in Grapheme-Colour Synesthesia. In Mattingley, J. B. \& Ward, J. (Ed.), Cognitive Neuroscience Perspective on Synaesthesia (pp. 281-288). Milano: Elsevier Srl. 